\title{
Beobachtungen über die Beschaffenheit des Zellkerns.
}

\author{
Von
}

\author{
Prof. Walther Flemming \\ in Kiel.
}

Hierzu 'Tafel XlıI.

In seinen "Untersuchungen über das Protoplasma " (3) beschreibt C. Heitzmann unter Anderem netzförmig angeordnete Fäden in den Kernen verschiedener Zellenarten. Aehnliches ist schon früher, und soviel ich finde, zuerst von Kleinenberg (1) am Kern des Hydraeies geschildert worden.

Im Anfang vorigen Jahres theilte ich Näheres über gerüstförmige Structuren in den Kernen des Eierstockseies von Muscheln und über die an ihren Kernkörpern wahrzunehmenden Erscheinungen ${ }^{1}$ ) mit (5). Die ersteren Angaben wurden wenige Monate später durch 0. Hertwig (9), und durch E. van Beneden (10) für die Eikerne von Echinodermen (Toxopneustes, Asteracanthion) und Säugethieren (Maus) bestätigt.

Als ich die erwähnten Beobachtungen gewann und zusammenstellte (Frühling und Sommer 1874), war mir der nähere Inhalt der ein Jahr vorher erschienenen Heitzmann'schen Arbeiten noch nicht bekannt geworden; auch von den beiden eben genannten Forschern ist er unberucksichtigt gelassen. Es ist dies vielleicht daraus erklärlich, dass Heitzmann durch die sehr kühnen und zum grossen Theil nicht hinreichend gestützten Hypothesen, in denen er die ganze Zellentheorie umzustossen strebt, eine minder günstige Kritik herausgefordert und damit auch für die exacten und werth-

1) Ich erlaube mir hier ein Missverständniss zu berichtigen, das in dem eben erschienenen Jahresbericht von H ofmann-Schwalbe, Allg. Anat. pag. 33, vorgefallen ist. Es wird dort berichtet, ich hätie den (grösseren) Nebentheil des zweibuckligen Kernkörpers gegen Essigsäure resistenter gefunden wie den (kleineren) Haupttheil. Ich habe im Gegentheil beschrieben, dass der Nebenthejl in Essigsäure viel stärker quillt wie der (aucb etärker tingirbare) Haupttheil. 
vollen Beobachtungen, die seine Arbeiten enthalten, die Aufmerksamkeit Anderer herabgestimmt hat. Diejenige, um die es sich hier handelt, verdient aber solche Aufmerksamkeit deshalb in besonderem Grade, weil sie sich nicht wie die übrigen erwähnten allein mit den Kernen einer Zellenart befasst, sondern das Gesehene als ein allgemeines Structurverhältniss des Kerns in Anspruch nimmt.

Seitdem und zum Theil gleichzeitig mit den beiden letzterwähnten, sind schon mehrfach einschlägige Befunde mitgetheilt worden : so besonders von Schw albe (8) über die Ganglienzellenkerne der Retina und anderer Orte. Frommann (7) hat Heitzmann's Beschreibung bezüglich der Kerne der Krebsblutzellen bestätigt. Eine Anzahl schon früherer Angaben und Abbildungen in Strasb u rg e r's Werk (6) betrifft offenbar dieselben Dinge, die hier behandelt sind; obwohl allerdings der Verfasser, so viel ich entnehmen kann, dieselben für vorübergehende Zustände hielt und annimmt, der Zellkern sei nzur Zeit seiner vollsten Wirksamkeit stets aus einer homogen glashellen Protoplasmamasse gebildet, in der weder Vacuolen noch Kernkörperchen zu beobachten “ (p. 254).

$\mathrm{Ob}$ manche von den Strukturen hierher gehören, die $\mathrm{M} \mathrm{a} \mathrm{y} \mathrm{zel(12)}$ aus in Theilung begriffenen Epithelkernen und in Beziehung zu der Theilung selbst beschreibt, kann ich zunächst nicht entscheiden und möchte es nur einstweilen vermuthen. Endlich hat auch Bütschli in seinem jüngst erschienenen schönen und umfangreichen Werk(14) Angaben über die Kerne der rothen Blutkörper gemacht, welche sich an die von Heitzmann anschliessen; und ebenso scheint mir hier anzuziehen, was derselbe Autor über die Kerne der Spermatozoenkeimzellen von Blatta orientalis aussagt (p. 39). - Vielleicht mag mir noch manche von anderer Seite stammende Einzelangabe entgangen sein; da es sich hier einstweilen nur um eine kurze Mittheilung handelt, die möglichst wenig durch Literatur beschwert werden soll, so erlaube ich mir das Suchen danach bis auf Weiteres zu verschieben.

Nachdem ich Heitzmann's Befunde verglichen hatte, und in Hertwig's und van Beneden's Angaben die Bestätigung fand, dass die Netze auch in den Kernen anderer Eizellen vorkommen bei Asteracanthion sind sie, wie ich mich hier überzeugte, noch leichter zu sehen wie bei den Najaden - schien es mir zunächst geboten, ein möglichst sicheres Urtheil zu gewinnen, ob das Gerüst im Kern wirklich eine intra vitam vorhandene Structur ist. Durch alles Vor- 
liegende wird diese Frage noch nicht entschieden. Allerdings sind meine Beobachtungen an den Eizellen von Unio und Anodonta am ganz frisch entnommenen $\mathrm{Ei}$ und ohne jede Zusatzflüssigkeit gewonnen, ebenso gewiss diejenigen 0 . Hertwig's und van Beneden's, die sich über die Lebenstreue der betreffenden Bilder auch weiter keine Scrupel gemacht zu haben scheinen. Es ist aber damit nicht ausgeschlossen, dass es sich schon um Leichenerscheinungen handeln könnte; es möchten ja schon mit dem Herausnehmen der Eizellen, mit ihrer Entfernung aus den intra vitam vorhandenen Diffusionsverhältnissen Gerinnungen im Kerninhalt erfolgen können; und ich habe dieser Möglichkeit am genannten Orte (p. 20) darum ausdrücklich ihr Recht gelassen, obschon ich sie als nicht gerade wahrscheinlich ansehen musste. - Der gleiche Einwand bleibt möglich gegen die Struktur der Knorpelzellenkerne, die Heitzmann nach dem frisch entnommenen Schnitt beschreibt; und würde hier um so gewichtiger sein, wenn man mit $\mathrm{H}$ eit $\mathrm{zmann}$ eine Canalisirung der Knorpelsubstanz annimmt, Dank welcher eine Einwirkung der Untersuchungsflüssigkeit (wenn schon Blutserum oder Kochsalzlösung, darum doch nicht sicher windifferent«) sehr erleichtert wäre, obschon ich die Annahme einer solchen Canalisirung nicht zu unterschreiben denke. Die von Heitzmann und Frommann ferner beschriebene Structur in den Krebsblutzellen tritt, nach der Verfasser Darstellung, erst auf, wenn sogar gewisse Veränderungen der Zelle selbst stattgefunden haben. Für die Kerne der rothen Blutzellen von Triton, in denen H. Körnchen mit einzelnen sie verbindenden Fäden wahrnahm, werden wir auf ein Unverändertbleiben auch im frischen Blutpräparat nicht schliessen dürfen, wenn wir bedenken, dass an einem solchen bekanntlich sogar oft Ortsverschiebungen der Kerne im Leibe der Blutzellen eintreten. Mit Berufung auf absolute Lebendigkeit des Objects kann von allen bisher vorliegenden wohl nur der eineBeobachtungsfall Heitzmann's ins Feld treten, der die Kerne lebender Amöben betrifft (p. 101); aber gerade diese, auch von Schwalbe in erster Hand citirte Angabe redet $\mathrm{nicht}$ von einer eigentlichen netz- oder gerüstartigen Structur im lebenden Kern, sondern nur von einzelnen Vacuolen in demselben, deren Vorkommen ja schon länger bekannt ist. - So betreffen auch Schwalbe's Untersuchungen zwar lebensfrische, aber doch nicht lebende Gewebe; Büts chli endlich, in dessen Abbildungen übrigens die bezüglichen Structuren keineswegs so deutlich zu er- 
kennen sind, wie es seiner Beschreibung und meinen Objecten entspricht, untersuchte die Blutkörper der Amphibien nach eigener Angabe gleich mit Essigsäurezusatz, und die einzige von ihm abgebildete rothe Blutzelle, bei welcher nach der Erklärung dies Reagens nicht angewendet war, zeigt im Kern auch keine Spur von dem Netzwerk.

Ich wandte mich also an das lebende Gewebe, indem ich ein Object benutzte, das durch die Grösse seiner klementartheile und ihrer Kerne besondere Chancen versprach, den Erdsalamander, und ein Gewebe, das für schonende Beobachtung im lebenden $\mathrm{Zu}-$ stand geeignet und dabei durchsichtiger ist, als Mesenterium und Lunge, die $\mathrm{Har} n$ blasenwand. Es wurde zunächst an curarisirten Thieren gearbeitet, was auch den Vortheil hat, dass sich bei solchen die Blase meist ziemlich stark gefüllt zeigt und keine Aufspritzung derselben nöthig ist. Eine derartige natürliche oder künstliche Ausdehnung der Blase ist für die Beobachtung nöthig, da sonst die Faltungen das Object viel zu sehr verdunkeln. Wo die Füllung der Blase nicht hinreichend durch die Vergiftung erzielt isț, kann man sie leicht durch Injection von schwacher Kochsalzlösung in die Cloake erzielen; dies verändert nichts am Gewebe der Wand, wie ja von vornherein anzunehmen sein wird und wie es auch der Vergleich der Bilder ergiebt. Doch habe ich sehr pralle Aufspritzungen vermieden, um nicht durch Zerrung etwa ein vorzeitiges Absterben zu veranlassen. Die Curarelösung wurde nie in die Bauchhöhle, sondern unter die Zunge eingespritzt. Ich habe ausserdem auch melrere Male an nicht vergifteten Thieren die Bauchhöhle rasch geöffnet, die hervorquellende Blase schleunig abgebunden, losgeschnitten und montirt, so dass zwischen dem Anschneiden der Bauchhöhle und dem Beginn der Beobachtung im günstigsten Fall nicht einmal mehr als etwa 10 Secunden verliefen. Der Vergleich mit den curarisirten Thieren zeigte, das in beiden Fällen ganz das Gleiche an den Gewebselementen zu sehen ist 1). - Die Blase wurde bei den curarisirten Salamandern auf einen planen auf den Objectträger gekitteten Glassockel neben das Thier gelagert und ein Deckglas horizontal, ohne weiteren Flüssigkeitszusatz, rasch auf ihre Kuppe gedeckt. Jüngere Thiere eignen sich besonders wegen der geringeren Fibrillen-

1) Diese Vorsicht wurde gebraucht, weil häufig - wenn auch keineswegs immer - nach der Curarvergiftung die Bauchhöhle stärker wie gewohnlich mit Flüssigkeit gefüllt ist; es wäre denkbar, dass durch einen solchen beginnenden Vergiftung-Ascites die Kerne schon verändert würden. 
menge. Da durch die dicke, oft nicht ganz klare Flüssigkeitsschicht, durch die untere Blasenwand und häufig im Innern vorhandene Schleimflocken Licht verloren geht, nimmt man am Besten den ganzen Blendungsapparat des Mikroskops heraus.

In den hier zu beschreibenden Verhältnissen ändert sich bei solcher Beobachtung stundenlang nichts. Will man für andere Zwecke längere Zeit und mit starken Immersionslinsen arbeiten, so empfehlen sich weitere Vorrichtungen, mit deren Beschreibung ich den Leser hier nicht aufhalte. Bei diesen Untersuchungen kam meistens uur Hartn. Syst. VIII, selten IX à imm. in Anwendung.

Man bemerkt bei höchster Einstellung die Kerne der Endothelzellen, vielfach aber nicht alle durch Grösse und runden oder nahezu runden Umfang ausgezeichnet, ziemlich blass; bei fast derselben Einstellung wenig tiefer, die feinen Fibrillen des Bindegewebes und dessen Zellenkerne, grösstentheils etwas kleiner wie die Endothelkerne, und vielfach länglich oder unregelmässig geformt, zum Theil auch rund; zugleich Muskelbündel, Gefässe und einzeln laufende Muskelzellen, letztere theils spindelig, theils drei- bis sechsfach verästelt (s. u.). Die Kerne der spindeligen sind stäbchenförmig oder lang-elliptisch, die der verästelten ebenso oder kurz-elliptisch, oder dreilappig. Bei ganz tiefer Einstellung endlich, schon undeutlicher, das Epithel. Selbstverständlich finden sich hic und da Leukocyten im Gewebe; endlich Kerne der Nervenfasern, nur hie und da für den, der Goldpräparate der Blase kennt, sicher zu diagnosticiren.

Bei hellem Licht von weissen Wolken oder bei sehr gutem künstlichen, wie das für die Wahrnehmung dieser Dinge absolut nöthig ist, sieht man nun in den Kernen der Endotholzellen. Bindesubstanz- und Nervenzellen und Muskelfasern vielfach völlig sicher zarte Gerüste, ganz ähnlich denen, die wir aus den Kernen der Eizellen beschrieben haben (Fig. 1). Sie sind nicht in allen Kernen wahrzunehmen, und wo sie es sind, nicht überall gleich deutlich; nur bei einer Minderzahl lässt sich das Gerüst ringsum in Zusammenhang mit der Keruwand finden. Das Wort Kernwand brauche ich, um auszudrücken, dass alle diese Kerne, ebenso die der Epithelien, eine scharf abgesetzte Wandschicht haben, ohne mich dabei hier auf die neuerdings erörterte Frage einzulassen, ob diese Wandschicht zum Kern oder zum Plasma der Zelle zu rechnen ist. Viele Kerne zeigen auf den ersten Blick nur eine verwaschene Zeichnung, die man ohne genaueres Nachsehen wohl in die Rubrik "granulirt" 
einschieben würde; sorgfältiges Einstellen und Sehen zeigt, dass viele der anscheinenden Granula optische Querschnitte von Bälkchen sind, die mit anderen zusammenhängen. Ein bis drei grössere Kernkörperchen sind an vielen, aber nicht an allen dieser lebenden Kerne wahrnehmbar, es kostet an vielen schon grosse Aufmerksamkeit, sie zu sehen. Sie sind niemals sehr scharf contourirt, am schärfsten noch an den Muskelkernen. Ausserdem sieht man vielfach noch kleinere blasse Granula, ebenfalls meist mit verwaschenen Umrissen, in den Balken des Gerüstes oder wo dies nicht deutlich zu sehen ist, anscheinend frei liegen; von optischen Querschnitten der Bälkchen sind sie nur durch ihre überwiegende Grösse und etwas abweichende Färbung oder Lichtbrechung unterschieden. An vielen Kernen ist von den grossen wie den kleinen Nucleolen mit bester Aufmerksamkeit nichts wahrzunehmen; dennoch sind sie vielfach wenigstens auch an solchen vorhanden, wic am besten die Chromsäurebehandlung lehrt. Wie gesagt. es kommen bald mehr, bald weniger Kerne vor, bei denen von all' diesen Dingen auch bei bestem Licht nichts zu erkennen ist; andere, bei denen Nucleolen, aber kein Gerüst wahrnehmbar ist. Dass es auch ihnen nicht fehle, dafür sprechen die weiter beschriebenen Reactionen.

Hin und wieder kann man auch an einem Kern einer weissen Blutzelle in einem Capillargefäss, oder einer Wanderzelle, wo eine solche gerade günstig dafür liegt, die Netzstructur erkennen, ebenso an fibrillenarmen Stellen auch wohl bei tiefer Einstellung in einem Epithelkern; doch sind das Ausnahmen, meistens sind die Epithelkerne und die Leukocytenkerne zu blass um etwas sehen zu lassen. Dasselbe gilt von den Kernen der rothen Blutzellen. Dennoch darf man auch allen diesen Kernen, nach dem Ergebniss der jetzt zu besprechenden Reactionen, dieselbe Beschafienheit zuschreiben wie den übrigen.

Wenn man unter das aufgehobene Deckglas rasch ein Tröpfchen schwache Essigsäure bringt ( $1 / 10$ pCt. genügt) und schnell wieder eindeckt, oder, mit ganz dem gleichen Erfolg, wenn man die eben herausgenommene Blase eines frisch angeschnittenen Thieres mit der Säure eindeckt und sofort beobachtet, so erscheint momentan in allen Kernen der Blasenwand, der Epithelien und der Blutzellen ein äusserst scharf ausgesprochenes, mit der Kernwand überall zusammenhängendes Gerüst. Kernkörper und Nebenkernkörper sind darin nur in nur in einzelnen Kernen, und niemals gerade sehr deut- 
lich sichtbar, was aber nur von der starken Lichtbrechungsfähigkeit der Bälkchen herrührt; denn wenn man deren Effect durch Glycerinaufhellung mildert (Fig. 2), so sieht man das Netz zarter, aber noch vollkommen deutlich, und in seinen Balken an vielen Kernen auf das Deutlichste die grösseren und kleineren Nucleolen.

Noch weit klarer kommt das Gerüst durch anderweite Reagentien und Tinctionen zur Anschauung. Es giebt gleichen Erfolg, ob man die gefüllte lebende Blase des curarisirten Thieres abbindet und gleich in die Flüssigkeiten einlegt, oder ob man sic am decapitirten Thier von der Cloake aus mit denselben injicirt und dann in den gleichen Lösungen liegen lässt, bis sie erstarrt ist. Das Epithel, wenn man will auch das Endothel, kann nach derartiger Behandlung mit Chromkali oder verdünntem Alkohol nachträglich abgepinselt werden, um die Details der Muskeln und der Bindesubstanz klarer zu haben; für die hier besprochenen Verhältnisse der Kerne ist aber Alles auch bei erhaltenem Epithel reichlich durchsichtig, die Kernstructur tritt sogar schärfer in Wasser hervor, wie in aufhellenden Flüssigkeiten.

An Chrom k a li präparaten (1-4p. c.), die mit Haematoxylingefärbtsind, tritt das Netzgerüst im Kern und die Wandschicht des letzteren in überraschender Schärfe und tiefblauer Farbe auf (Fig. 4, 5, 6), während die in seinen Lücken vorhandene Substanz des Kernes blasser blau, aber immerhin viel dunkler gefärbt ist, wie das Plasma der Epithel- und sonstigen Zellen und dic Muskeln. - Die Netze sind - und das gilt auch für alle folgenden Reagentien - richt in allen Kernen gleich dicht angeordnet. In der blassblau gefärbten Zwischensubstanz des Netzes zeigen sich stellenweise kleine helle, ganz ungefärbte Lücken ${ }^{1}$ ). Der Zusammenhang der Netze mit der Kernwand ist nicht bei allen Kernen deutlich, bei vielen aber zweifellos, - Die Hauptnucleolen (so nenne ich die grösseren) treten bei dieser Behandlung nicht durch schärfere Färbung hervor, die Nebennucleolen sind sogar zum grössten Theil in den scharfgefärbten Netzbälkchen gar nicht wahrnehmbar. Deutlicher sind erstere, und wenigstens etwas besser sichtbar ist ein Theil der Letzteren an ungefärbten Objecten aus Chromkali. Diese Undeutlichkeit der Kernkörper ist deshalb nicht etwa darauf zu beziehen, dass sie durch worden.

1) Sie sind in der Figur 5 und 6 boim Stich nicht mit dargestellt 
das Chromkali zerstört wurden. Ebensowenig ist daran zu denken, dass etwa an den Blasen, die diese Bilder geben, die Kernkörper überhaupt fehlten oder besonders undeutlich wären: denn wenn man von einer mit Kochsalz aufgespritzten Blase die eine Hälfte in Chromkali, die andere in dïune Chromsäure (s. u.) einlegt, so sieht man an letzterer die Kernkörper ausgezeichnet, an ersterer nach Färbung undeutlich oder gar nicht. Die Sache kann also nur dahin gedeutet werden, dass das Chronkali die Balken des Netzes sehr stark lichtbrechend macht, die Nucleolen aber nicht; während die Chromsäure in dieser Richtung auf Beide in ziemlich gleicher Weise wirkt.

Carm in tinction von Chromkalipräparaten zeigt der Form nach ganz dasselbe wie Blauholzpräparate, aber $n$ icht stets der Färbung nach. Wührend nämlich die Interreticularsubstanz durch das Carmin gleichmässig rosenroth gefärbt ist, hat das Netzwerk an Präparaten, die aus starken Lösungen des Chromsalzes kamen, ganz seine blassgelbe Chromkalifarbe behalten (Fig. 7 ab, es ist hier, um Colorirung zu sparen, hell dargestellt) und tritt in dieser scharf gegen jene hervor. Die Nucleolen sind dabei ebensowenig deutlich wie mit Hämatoxylin. Doch kann man sie bei besonderer Behandlung deutlich machen und durch stärkere Tinction gefärbt darstellen.

Die Verdeutlichung der Netze durch Chromkali erfolgt in dem eben beschriebenen, hohen Grade nur, wenn man Lösungen von mindestens $1 \mathrm{pCt}$. benutzt. Bei geringerer Concentration stellen sich die Gerüste undeutlicher dar.

Alkoholbehandlung liefert ebenfalls ganz deutliche Netze von entsprechender Anordnuvg, wie die des Chromkali. Meistens erscheinen aber die Balken etwas dicker und unregelmässiger geformt wie nach jener Beliandlung; was ebenso für die Chromsäure gilt. Die Nucleolen sind in den meisten Fällen mit Alkohol etwas deutlicher wie mit Chromkali.

Die Chromsäure unterscheidet sich, wie erwähnt, dadurch in ihrer Wirkung von den beiden vorigen Reagentien, dass sie die Haupt- und Nebennucleolen äusserst scharf sichtbar zeigt, und zwar sowohl ohne als mit nachfolgender Tinction (Fig. 8). Man überzeugt sich hier aufs Deutlichste, dass die Nucleolen in den Bälkchen liegen. Die grösseren Kernkörper sehen nur selten rund aus, sondern sind meist von unregrelmässigen, rauhen Contouren, oft wie aus mehreren Körnern zusammengeballt; dasselbe kann man übrigens 
wo sie überhaupt zu sehen sind, oft auch an Alkohol- und Chromkaliobjecten bemerken. Ohne Färbung glänzen sie an den Chromsäurepräparaten weisslich; mit Carmin tingiren sie sich rosenroth, nicht erheblich stärker wie die Kernwand und das Netz, während an Alkohol-Carminpräparaten ihre Färbung bedeutender zu sein ptlegt.

$\mathrm{Zu}$ bemerken bleibt für alle diese Behandlungen und Tinctionen, dass das Gerüst nicht an allen Kernen gleich stark lichtbrechend am ungefärbten, noch gleich stark tingirt am gefärbten Objektist. Es kommen vielmehr - am auffälligsten bei den intensiven Blauholztinction - alle möglichen Uebergangsstufen von blassen Bildern zu den schärfstgezeichneten vor; und zwar nicht etwa gruppenweise vertheilt, so dass eine ungleichmässige Einwirkung der Grund sein könnte, sondern bunt durch einander an Kernen, die dicht beisammen liegen; auch sind nicht etwa die Kerne einer Art von Gewebstheilen, der Endothelien, Muskeln, Bindesubstanzzellen, bevorzugt für blassere oder schärfere Netze, sondern es kann zum Beispiel ein Muskelund ein Lndothelkern mit scharfblauen Netzen neben einem andern Muskel- und Endothelkern mit ganz matt-blassgrauen gefunden werden (Fig. 4). Man wird sich bei diesem Verhalten wohl ohne Weiteres erinnern, dass am lebenden Object auch die Structuren nicht in allen Kernen gleich deutlich sind, und den Wahrscheinlichkeitsschluss ziehen, dass die Gerüste nicht in jedem Kern und nicht in jedem gegebenen Moment überall die gleiche Beschaffenheit haben werden, sondern dass sie etwas Physiologisch-wechselndes sind.

Anders, wie die besprochenen Reagentien, verhält sich die $0 \mathrm{~s}$ miumsäure. Man sieht nach ihrer Anwendung in den Kernen selten mehr, meistens aber noch weniger von den Gerüsten wie am frischen Gewebe; nachfolgende Picrocarminfärbung lässt noch weniger erkennen. Der Kern erscheint dabei fast gleichmässig rosenroth, nur mit starken Systemen kann man in ihm verwaschene Andeutungen von netzförmiger Zeichnung ausmachen ${ }^{1}$ ). Die Kernkörper dagegen und Nebennucleolen sind sehr deutlich, doch nicht intensiver roth gefärbt wie die übrige Substanz des Kerns, oft gar nicht geröthet, sondern gelb.

Sehr seltsame Eigenschaften der Kerngerüste, welche von allen

1) Bis jetzt wurde übrigens bloss $s \mathrm{chwächere} \mathrm{Osmiımsäure} \mathrm{(bis}$ $1 / 2$ pCt.) probirt. 
den erwähnten Reagentien verborgen gelassen werden, zeigt das Anilin.

Vielleicht wird es Anderen, die mit der schönen von Herman n angegebenen Anilinfärbungsweise ${ }^{1}$ ) gearbeitet haben, schon ebenso wie mir aufgefallen sein, dass man bei diesen Färbungen, die bei richtiger Anwendung nur die Kerne betreffen, in diesen nicht nur die grösseren eigentlichen Kernkörperchen stark gefärbt findet, so wie es die Carminund die reine Pikrocarminbehandlung leistet; sondern ein grosser, und zwar gewöhnlich der bei Weitem grösseste Theil der Kerne zeigt statt dessen eine Menge anscheinend discreter, oft verschieden stark gefärbter und verschieden grosser, rother Flecke. An unserem Object nun, mit seinen vershältnissmässig colossalen Kernen, lässt sich wahrnehmen, dass diese gefärbten Stellen nicht discret sind, sondern stärker tingirte Stellen im Netzwerk selbst darstellen, dessen blassere, aber doch noch leicht gefärbte Zwischenbalken sich auch nach der Nelkenölaufhellung noch recht gut unterscheiden lassen (Fig. 9). Ein Theil dieser stark gefärbten Stellen - ich will sie hier einmal kurz Anilinflecke nennen - ist offenbar identisch mit dem oder den Hauptkernkörpern und den Nebenkernkörpern. Aber das kann keineswegs für alle gelten: denn erstens ist ihre Zahl dafür viel zu gross - man müsste sonst schon sagen, dass das frische Object, die Essigsäure, Chromsäure und das Carmin einen grossen Theil der Kernkörperchen nicht sichtbar werden lässt, während das Anilin dies thut; zweitens sind die Anilinflecken vielfach nicht scharf abgegrenzt, keine eigentlichen "Körperchen ", sondern oft so beschaffen, dass eine grössere Partie des Netzwerks durch und durch stärker gefärbt ist, wie die angrenzenden.

Es zeigt also, kurz ausgedrückt, das Anilin eine anders beschaffene und weiter gehende Differenzirung im Kerninhalt, wie alle die anderen Behandlungsweisen.

Man könnte nun denken, und ich habe zunächst daran gedacht, dass diese Bilder Artefacte seien, dadurch bedingt, dass in Folge der Behandlung mit Anilin und Nelkenöl Zerreissungen und Zusammenballungen in dem Netzwerk stattfinden, und die Anilinflecke solchen dann stärker gefärbten Zusammenballungen entsprechen könnten; obschon dies sonderbar genug wäre, da ja das Präparat vorher in Alkohol absolut gehärtet ist, und vor der Färbung das

1) Tageblatt der Grazer Naturf.-Vers. 1875. 
Netzwerk ganz regelmässig zeigt. Jener Verdacht muss aber aus geschlossen werden. Er wird es erstens schon durch folgenden Versuch: Wenn man ein aufgehelltes Anilinpräparat, das die eben beschriebenen Dinge zeigt (Fig. 9), wieder in Alkohol, dann in Wasser bringt und darauf mit schwacher Essigsäure das Anilin auszieht, so sieht man nichts mehr von den vielen, vorher different gefärbten Stellen im Kern, sondern das Netzwerk erscheint ganz vollständig, gerade wie an einem Alkoholpräparat, das man bloss mit Essigsäure behandelt hat. An dem Netzwerk war also durch die Anilinbehandlung nichts zerstört worden. Geradezu sehlagend scheint mir ferner ein Umstand, auf den ich durch Präparate des Herrn E. Fischer aufmerksam gemacht wurde. Die Kerne in der äusseren Körnerschicht der Retina zeigen bekanntlich eine räthselhafte $Q u$ er scheibenschichtung. An einem $\mathrm{Hermann}$ 'schen Anilinpräparat sind nun ganz regelmässig diese Schichten abwechselnd die einen crass gefärbt, die anderen ungefärbt (Fig. 10). Wenn man die Querschichtung der äusseren Körner für Natur halten darf und das thut man ja allgemein, da sie auch am frischen Object zu sehen ist, und da sie eine, diesen Kernen eigene Specialität bildet - so wird auch dasselbe für die Stellen des Kernnetzes gelten können, die hier in unserem Falle durch das Anilin gefärbt blieben und man wird demnach sagen können: Es existiren hier in dem Netzgerüst der Kerne, ausser den auch sonst darstellbaren Nucleolen, noch Stellen von differenter Beschaffenheit, welche sich der Wirkung der übrigen erwähnten Reagentien entzieht, durch das Aniliudagegen zum Ausdruck gebracht wird ${ }^{1}$ ).

1) Ich brauche wohl kaum darauf hinzuweisen, dass man bei der Hermann'schen Behandlung durch allzu langes Ausziehen mit Alkohol auch die Anilinflecke zum Verschwinden bringen und alles blass machen kann. Dies geschieht offenbar bei dem einen Kern eher wie bei dem andern, und wieder bei dem einen Anilinfleck in je einem Kern eher, wie beim anderen. Daher die vielen verschiedenartigen Bilder auch dort, wo die Flecken in vielen Kernen deutlich und zahlreich sind: in anderen sind sie bei dieser Einwirkung schon seltener und blasser, in noch anderen nur die grösseren und kleineren Nucleolen mehr gefärbt, an denen der Farbstoff besonders lange haftet. Natürlich kann man trotzdem auf eine differente Beschaffenheit der Stellen schliessen, denen die Anilinflecke entsprechen: denn wenn eine Stelle den Farbstoff länger hält wie eine andere, so wird sie anch anders beschaffen sein. 
Ich bemerke nocb, dass an vielen, doch nur der Minderzahl der mit Reagentien behandelten Kerne Vacuolen verschiedener Grösse vorkommen, die dann das Netz und seine Zwischensubstanz gleichmässig unterbrechen. Auch an lebenden Kernen sind solche manchmal wahrzunehmen.

Schliesslich sei noch kurz der Wirkung des destillirten Wassers gedacht: es zerstört sehr rasch die Gerüste und Nucleolen, macht den Kern aufquellen und lässt nur diffuse granulirte Massen, an der Membran angehäuft, in ihm erkennen. Im Wesentlichen wird sich dies mit Auerbachs Ergebnissen in Einklang stellen.

Damit breche ich vorläufig den Bericht über diese Versuche $a b$, deren Fortsetzung mich weiter beschäftigen wird, und setze nur noch hinzu, dass ich zugleich auch auf Kerne verschiedener anderer Gewebstheile geachtet, und an ihnen vollkommen mit dem hier Beschriebenen übereinstimmende Dinge gefunden habe. In den Zellkernen des hyalinen Knorpels von Salamandra z. B. sieht man am frisch entnommenen Schnitt, ohne Zusatz, die Gerüste in vollkommener Deutlichkeit, womit ich also den Befund Heitz mann's am Säugethierknorpel bestätigen kann. An Essigsäure- oder Chrompräparaten von $\mathrm{Mund-}$ und Riechepithelzellen, und Bindegewebszellen verschiedener Orte, sehe ich ferner bei Salamandra und Rana die Fasergerüste im Kerne auf den ersten Blick; doch ist es erklärlich genug, dass die Beobachter beim Frosch und Säugethier, auf Grund der hier kleineren Dimensionen, stets nur den Eindruck von Körnern, statt von Fasern erhalten haben und dass sich demnach dic landläufige Ansicht gebildet hat, die Essigsäure und die Chromsalze "machten die Kerne granulirt" ${ }^{1}$ ).

Ich stelle nun schliesslich die Frage: sind die Gerüste in den Kernen intra vitam verhanden, oder sind sie Gerinnungen, Leichenerscheinungen, Artefacte? - Dass die Netze der frischen Kerne

1) An Macerationspräparaten von Epithel aus schwächerem Chromkali wird der Zusammenhang des Gerüstes im Kern mit der Kernwand gewöhnlich nicht gefunden. So erklärt es sich wohl, dass Newell Martin (Journ. of anat. and phys. 8, Nov. 1873) von den Kernen der Riechzellen angiebt, sie seien stark granulirt und durch einen bellen Saum getrennt von einer homogenen Kapsel, die mit dem Zellkörper zusammen hänge. Dies ist die Kernwand. - Sehr schwacheChromkalilösungen zeigen überhaupt die Netze undentlich oder gar nicht. 
(Fig. 1) und die mit Reagentien dargestellten (Fig. 2, 4 u. a.) der Ausdruck identischer Dinge sind, wird dem Beobachter Beider nicht zweifelhaft bleiben. Es kann sich also nur darum handeln, ob die in den Kernen der lebenden Blasenwand, dicht neben durchströmten Blutgefässen sichtbaren Gerüste nicht schon Gerinnungen, ob die betreffenden Kerne nicht schon im Absterben begriffen sind. Man könnte sich dagegen darauf berufen, dass verschiedene der oben citirten Beobachter ähnliche Bilder, wenn sie auch an Gewebstheilen vorkamen, die dem lebenden Körper abgeschnitten oder entnommen waren, dennoch ohne Weiteres für gegebene Structuren gehalten gehaben. Doch das ist noch kein Grund, ebenso zu verfahren; und da ich nicht den Schein einer Vorliebe für die Präexistenz der Kernnetze auf mich laden möchte, so gebe ich ausdrücklich noch immer die Möglichkeit zu, dass die Kerne durch das rasche Herausholen der Blase und das Auflegen des Deckglases schon getödtet oder verändert, und die Netze Gerinnungen sein könnten. Ein endgültiger Entscheid wird sich wohl bald an geeignetem Object gewinnen lassen; vor der Hand, wo es mir lediglich darauf ankommt, auf diese Dinge aufmerksam zu machen, will ich nur bekennen, dass mir diese Annahme aus folgenden Gründen sehr unwahrscheinlich vorkommt: erstens wegen der Form und Anordnung der Netze. Es ist wahr, dass sie nicht in allen Kernen gleich dicht sind, und dass ihre Balken nach Anwendung der einen oder andern Reagentien bald etwas schlanker, bald dicker, bald mehr weniger regelmässig contourirt sind. Aber diese Abweichungen bleiben doch in sehr mässigen Grenzen und lassen sich in diesen sehr wohl begreifen, wenn man eine nicht für jeden Zustand des Kernes gleiche Beschaffenheit seiner Substanz, und eine etwas differirende Wirkung der einzelnen Reagentien annimmt. Wäre dagegen das Ganze nichts anderes als ein Gerinnsel oder die stehengebliebenen Theile verfestigter Substanz zwischen confluirten, künstlichen Vacuolen, so würde man eine viel unregelmässigere Anordnung der Netze erwarten, bald sehr locker, bald sehr enge, gerade wie bei anderen in Netz- oder Gerüstform gerinnenden Substanzen. In dem Blutplasma, das die Gefässe der lebend abgebundenen Blase füllte, kann man an Chrompräparaten die schönsten Gerinnungsnetze des Fibrins finden. Aber diese sind bald locker, bald eng (nie wohl übrigens so eng, wie die Kernnetze), bald endlich fehlen sie ganz. Wären die Kernnetze Gerinnsel, warum zeigen sie dann nicht 
auch solche Varianten und warum gerinnt die Masse hier nicht auch oft homogen, statt netzartig, wie das Fibrin es thut?

Zw eitens kommt die constante Lage der Kernkörper in den Balken der Gerüste in Betracht. Bei Gerinnungen würde zu fragen sein, warum dieselben gerade immer, auch wo die Netze lockerer sind, in den Fäden des Gerinnsels eingeschlossen werden müssten.

Drittens, die Bilder der Anilinfärbung. Es ist schwer einzusehen, warum die Balken eines blosen Gerinnsels sich an gewissen Stellen anders, wie an anderen verhalten sollten.

Aus all' diesen Gründen verliert die Annahme, dass die Netze Gerinnungen darstellen, an Wahrscheinlichkeit und dürfte, so lange nicht wenigstens irgend welche weitere Belege für sie zu finden sind, zurücktreten müssen gegen die andere: dass die Netze ein gegebenes Structurverhältniss des Kerns, oder um vorsichtiger zu reden, der Ausdruck eines solchen sind.

Denn diese verallgemeinernde Ausdrucksweise darf man sich wohl erlauben. Es wird schwerlich Jemand glauben, dass, was an den Kernen all' dieser verschiedenen Gewebselemente von Amphibien, Säugethieren, Mollusken und Echinodermen, endlich bei Pflanzenkernen vorkommt, nicht eine allgemeine Eigenschaft des Kernes zu nennen sei. Damit soll nicht gesagt sein, dass es nicht Kerne geben kann ohne solche Structur; denn wenn wir dieselbe als existent annehmen können, so wissen wir doch nicht ob sie jedem physiologischen Zustande des Kernes zukommt.

Das Gerüst, vorausgesetzt dass es präexistirt, hat ferner noch eine differente Structur in sich selbst. Erstens liegen in ihm die Kernkörper und Nebenkernkörper, als besonders beschaffene Theile seiner Balken. Zweitens lassen die Anilinbilder schliessen, dass nicht alle Partien des Netzwerks von gleicher chemischer, vielleicht also auch nicht von gleicher morphologischer Beschaffenheit sein werden. Wenigstens scheint mir die Annahme solcher Structurdifferenzen zunächst die betreffenden Tinctionserscheinungen am Einfachsten zu erklären.

Wenn ich hier den Ausdruck Struct ur brauche, so mag wiederholt sein, dass damit nicht ein Bau gemeint sein muss, der dem Kern von Anfang bis Ende seines Bestehens anhaftet, wie dem Wirbelthier das Skelett, sondern eine Difierenzirung der Substanz, die möglicherweise physiologisch wechseln und verschoben werden kann; die aber, soweit sie constant wiederkehrt, darum nicht min- 
der morphologisch festgestellt und bezeichnet $\mathrm{zu}$ werden verdient. Ich mache diese Anmerkung besonders gegenüber einer Stelle bei Bütschli. Derselbe sieht zwar die Radienstructur in sich theilenden Zellen nicht mit A u erbach als blossen Ausdruck von "Kernsaftströmchen « an, kann aber auch nicht mit mir "dieselbe auf ein gegebenes ${ }^{1)}$ Structurverhältniss des Plasma beziehen (, da man „im Plasma, dessen Theilchen ihre gegenseitige Lage beständig zu wechseln fähig $\operatorname{sind}^{2}$ ), von Structurverhältnissen im gewöhnlichen Sinne nicht reden könne . (!) Warum nicht? Es gibt ja viele Bauten die nicht für die Ewigkeit, sondern temporär errichtet werden. Vielleicht ist Bütschli inzwischen schon durch die Mittheilungen Kupffer's (13) über die Structur des Plasma in Speicheldrüsen- und Leberzellen in jener Ansicht erschüttert worden. Wo nicht, so stehe ich trotzdem nicht davon ab, alle optisch wahrnehmbaren Dinge im Plasma, ob sie nun bleibend oder vorübergehend sind, Bauverhältnisse oder Structuren zu nennen, sofern sie sich in irgend einer bestimmt wiederkehrenden Form und Anordnung zeigen. Jede Structur ist ja im Grunde nichts Anderes, als Substanzdifferenz.

Das hier Mitgetheilte giebt einen Hinweis darauf, dass man in der Forschung nach der Morphologie des Kerns noch hinlänglich zu thun hat, und dass also die schönen Arbeiten Auerbachs, in welchen diese Structuren bisher keine Erwähnung gefunden haben, in keiner Richtung als abschliessend angesehen werden können. Darin wird man wohl keinen Mangel an Würdigung für Auerbachs Studien und für die vielen wichtigen Aufschlüsse sehen wollen, die wir denselben schon verdanken. Es ist selbstverständ-

1) Meine betreffenden Worte lauten (l. c. p. 115): seine in diesem $\mathrm{Zustand}$ der Cytode gegebene radiäre Structurc. Bütschli wird mir, indem er die gesperrten Worte auslässt, wohl nicht die Meinung unterschieben wollen, dass die Radienstructur immer in Plasma angelegt sei, da ich ja grade beschrieben habe, wie sie während der Theilungen auftritt und schwindet.

2) Wenn hier unter sTheilchen \& die Atome oder Moleküle der Plasma verstanden sein sollen, so gilt die Fähigkeit zur Lageveränderung nicht nur für sie, sondern für die aller Körper, da alle chemischer Umsetzungen fähig sind. Sollten aber gröbere Massentheilchen gemeint seix, so darf man wohl fragen, woher denn der Verfasser weiss, dass solche beständige Bewegungsfähigkeit der Theilchen sdem Plasma» als Eigenschaft zukommt. 
lich, dass Verhältnisse, die bei ihrer Zartheit im frischen Zustand selbst in den grossen Kernen des Erdsalamanders nur mit grosser Sorgfalt zu sehen sind, bei der Benutzung der Objeste entgehen konnten oder mussten, die Auerbach verwendete und die für das Erkennen dieser Structuren grösstentheils nicht günstig sind. Freilich ist aber auch klar, dass, wenn sich die Richtigkeit des hier als wahrscheinlich angesehenen Kernbaues bestätigt, viele von A u e rbach's Beobachtungen eine andere Deutung erfahren müssen, als er ihnen gegeben hat. Vor der Hand möchte ich es für annehmbar halten, dass die "Zwischenkörner" A u erbach's hauptsächlich optischen Querschnitten der Balken des Netzwerks entsprechen mögen, dessen Continuität an vielen seiner Objecte $-z$. B. den Leberzellenkernen der Fische - im frischen Zustand gewiss schwer erkennbar sein wird. Von einer "beweglich-schwebenden " Suspendirung der "Zwischenkörner" und der Nucleolen aber in einem flüssigen Kerninhalt, wie sie Auerbach annimmt, muss selbstverständlich abgesehen werden, wenn diese Körner und Nucleolen Theile, resp. Einlagerungen eines den Kern durchsetzenden Gerüstes sind.

Es ergiebt sich wohl überhaupt, dass es zur Zeit sich eher empfiehlt über die feinere Morphologie und Chemie des Kerns noch recht viele Erfahrungen zu sammeln und zu sichten, als bereits umfassende Theorien über sein morphologisches Wesen aufzustellen, wie das in vieler Hinsicht schon von $A$ uerbach, und ganz neuerdings von Richard Hertwig (15) versucht ist. Jede neu gefundene Thatsache kann sonst im Stande sein, einer solchen Theorie ganz oder zum Theil den Boden wegzuziehen. So scheint es mir z. B. in diesem Fall zu sein mit der neinheitlichen Auffassung der verschiedenen Kernformen", die R. Hertwig a. a. O. formulirt hat, unstreitig nach besten und vortrefflichen Kenntnissen und nach eigener ausgedehnter Beobachtung; darum, wenn ich mir hier unter Vergleichung des Beschriebenen eine Kritik dieses Versuchs erlaube, so richtet sie sich auch nicht gegen die Art, wie derselbe ausgeführt ist, sondern dagegen, dass er überhaupt gemacht wurde, ehe denn es - nach meiner Ansicht - Zeit war.

Die Sätze R. Hertwigs lauten:

1) Das Wichtigste am Kern und das für ihn Charakteristische ist die -Kernsubstanz«, ein Eiweisskörper, welcher, wenn er auch viel Aehnlichkeit mit dem Protoplasma hat, sich doch durch zahlreiche Eigenthümlichkeiten von ihm unterscheidet. 
2) Die Kernsubstanz ist, bei den einzelnen Kernen in verschiedenem Maasse, von einer Flässigkeit, dem sKe rnsafte durchtränkt.

3) Die primitiven Kerne sind nichts als nackte Klümpchen dieser Kernsubstanz (Kerne des reifen und befruchteten Eies, der Furchungszellen etc.)

4) Aus diesen primitiven Kernformen leiten sich die übrigen durch folgende Differenzirung $a b$ :

a) indem sich eine Kernmembran entwickelt (Kerne der Infusorien);

b) indem sich der Kernsaft und die eigentliche Kernsubstanz sondern, wobei dann der Kernsaft

a) unregelmässig im Kerne vertheilt wird und zahlreiche Vacuolen bildet,

B) sich zwischen Kernmembran und Kernsubstanz ausbreitet und so die Bildung von Kernkörperchen veranlasst (bei den meisten pflanzlichen und thierischen Zellen).

c) Indem ein ernährendes Protoplasmanetz durch die Poren der Membran in die Kernhöhle eindringt und den vom Kernsaft erfüllten Hohlraum durchsetzt.

Den ersten dieser Sätze wird wohl jeder neuere Histiolog unterschreiben; wenn nicht $A$ u erbach, der früher die Kernkörperchen mit der Substanz junger Zellen identisch nannte, noch an dieser Ansicht festhalten sollte.

Der zweite Satz ist ebenso unbestreitbar und ebenso wenig neu, in so fern wir uns ja alle lebenden Eiweisskörper und eiweissartigen Körper von Flüssigkeit durchtränkt oder darin aufgequollen vorstellen müssen, und diese Flüssigkeit für besondere Gewebstheile auch gewiss vielfach eine besondere sein wird.

Der dritte Satz ist schwerlich haltbar. Wenn wir anzunehmen haben, dass in den Kernen einer Menge von Zellenarten, deren Inhalt man bisher, abgesehen von den Nucleolen, entweder für "klar" oder für "homogen " oder für "granulirt" gehalten hat, eine gerüstförmige Differenzirung der Substanz vorhanden ist, wenn aber diese Beschaffenheit sich bis jetzt nur an besonder günstigen, durch Grösse ausgezeichneten Objecten wirklich sehen lässt: so wird man gewiss nicht behaupten dürfen, dass die zarten kleinen »primitiven Kerne « nicht ähnliche oder andere Bauverhältnisse besitzen, von denen bisher Niemand etwas gesehen hat 1).

1) Beobachtungen, die ich kürzlich auf Sylt über die Entwickelung von Echinus miliaris machte, geben mir hierfür gerade noch einen Beleg in in die Hand. R. Hertwig betrachtet den centralen Pronucleus (Eikern n. o. Hertwig) der vor der Theilung stehenden Eizelle als besonders typisches Beispiel eines structurlosen primitiven Kerns. Bei Echinus zeigt nun der 
Zu 4): Dass sich Kernmembranen entwickeln, wie bei den Infusorien, so bei den weitaus meisten Zellen, ist bekannt. Was aber die folgenden Punkte angeht, so wird uns eine derartige Eintheilung der Kerne nach Vertheilung von Kernsubstanz und Kernsaft wenig befriedigen können, wenn wir wissen, dass z. B. in der Substanz, die H. sub 4 b $\beta$ als Kernsaft ansieht, noch ein gerüstartiger Bau vorhanden ist. Vielleicht können wir dahin übereinkommen, den Narnen Kernsubstanz und Kernsaft auf die Substanz des Gerüstes und dessen Zwischensubstanz zu übertragen; doch ist für den letzteren Fall einige Vorsicht nöthig, da man doch nicht weiss ob die Substanz auch wirklich überall flüssig ist.

Besonders aber hat mich der letzte Punkt überrascht. Hertwig, der die Gerüste aus eigener Anschauung nur am Kern des Seeigel- und Froscheies kennen gelernt hat, ist erstens der Meinung, dass ihr Vorkommen auf whoch differenzirte Kerne beschränkt sei“ - dies wiederlegt sich wohl durch das hier Mitgetheilte, oder wir müssten denn die meisten Kerne des Wirbelthierkörpers ebenfalls hoch differenzirt nennen. Denn an der Präexistenz der Netze in ihnen zu zweifeln ist Hertwig gewiss nicht in der Lage, nachdem er die Netze in den Eizellenkernen, also von nicht mehr sicher lebendigen Präparaten, doch ohne Bedenken für natürliche Structur genommen hat. Zweitens nimmt Hertwig ganz ernstlich an, dass das Netzwerk von aussen "durch die Poren der Kernmembran " in den Kern hineingewachsen sei (p. 77, 78-79), und hier eine ernährende Function ausübe. Wenn hieran zu denken wäre, so würde dasselbe auch für die übrigen hier beschriebenen Kernarten zu gelten haben und der Verfasser käme damit in eine ziemliche Uebereinstimmung mit Heitzmann, welcher überall ein durch die Kernwand hindurch zusammenhängendes "Protoplasmanetz in Zelle und Kern annimmt.

centrale Pronucleus nach Chromsäure- und Chromkalibehandlung nicht nur eine Anzahl von Körpern in seinem Innełn, die an den Pronuclei anderer Eier übrigens schon von Anderen (Auerbach, van Beneden) dargestellt wurden, sondern auch Netzfäden, die diese Körper verbinden. Am frischen Object sieht man sie freilich nicht mit hinreichender Deutlichkeit; und Oscar Hertwig, der die betr. Kernkörper an Chrompräparaten vom ToxopneustesEi ebenfalls erwähnt (p. 65), betrachtet sie als Artefacte. Ich sehe aber nicht ein, weshalb die Resultate der Chromwirkung gerade an diesem Object Kunstproducte sein sollen, während dieselbe z. B. an den Eierstockseiern dieselben Netze, die hier auch frisch zu sehen sind, getreu und nur verschärft sichtbar macht. 
Netzartige Anordnungen nun im Plas ma der Blasen ep ithelzellen finde ich an den mit Reagentien behandelten Objecten, deren KerneGegenstand dieser Abhandlung sind, allerdings in der deutlichsten Weise, und habe begonnen, mit Rücksicht auf $\mathrm{H}$ eitz $\mathrm{m}$ a nn 's Angaben sowie besonders die Befunde Kupffers an Drüsenepithelien, diesen Verhältnissen weiter nachzugehen. Ich kann aber vorläufig nur aussagen, das̀s ich mich trotz der Grösse derObjecte bis jetzt weder von "Poren" in der Kernwandung noch von einem Zusammenhang von Balken im Plasma mit den Balken des Kernes habe überzeugen können; wozu nebenbei noch bemerkt sein soll, dass die Netze im Plasma und die im Kern bei gleicher Behandlung sehr verschieden sich verhalten. An anderen Zellen sind die Netzbilder $n i c h t$ in der Art vorhanden, wie an den Epithelien. Jedenfalls vermag ich an den wohlerhaltensten Kernen, die die dicksten, deutlichsten Membranen zeigen, an deren Profil nicht die Spur von Lücken oder Poren wahrzunehmen. Ohne die Ansichten Heitzmann's über die allgemeine netzförmige Struktur des Protoplasma angreifen zu wollen, vielmehr vorläufig in dem Glauben, dass viel Wahres daran sein mag, ohne aber auch bis jetzt behaupten zu können, dass die eben erwähnten Netze im Epithelplasma Natur und nicht Kunst seinen - muss ich doch sagen, dass mir nach Allem was ich gesehen, ein Zusammenhang der Kernnetze mit Dingen ausserhalb des Kernes noch durch nichts erwiesen zu sein scheint.

In Hertwigs Aufsatz finde ich auf alle Fälle nichts, wodurch ein solcher Nachweis geführt wird; und unter diesen Umständen scheint mir die Hypothese, wdass ein ernährendes Protoplasmanetz durch die Membran in die Kernhöhle eindränge«, ganz in der Luft zu stehen. - Ich habe Anlass, den Verfasser für diesen kleinen Angriff um Entschuldigung zu bitten, da er selbst in bescheidenster Weise seinen Definitionsversuch nichts Anderes nennt, als eine $\mathrm{Zu}$ sammenfassung bekannter Thatsachen unter einem allgemeinen Gesichtspunkte. Ich opponirte, weil mir die Thatsachen, die ich als solche zugeben kann, zu bekannt scheinen, um noch einer Anführung zu bedürfen, und weil ich für alles Uebrige der Meinung bin, dass es sich eben noch nicht so rasch unter einheitlichen Gesichtspunkt bringen liess; vor Allem aber weil das Ganze ein passendes Beispiel dafür bietet, dass die besten Beobachter irre gehen können, sobald sie sich auf frühzeitiges Schematisiren einlassen.

Das Wort "protoplasmatisch", das R. Her twig als Attribut des 
Netzwerks benutzt und das Oscar Hertwig (p. 6) auch mir zuzuwenden scheint, habe ich ebenso wenig in Beziehung zu den Gerüsten der Kerne, als auch sonst je im Ernst gebraucht und halte es hier für höchst unglücklich, eben weil der Missverstand dadurch entstehen konnte, als handele es sich nicht um einen Theil des Kernes, sondern um etwas Hereingewachsenes. Wie ich denn überhaupt in dem Ausdruck "protoplasmatisch" irgend etwas Definirendes oder sonst Beglückendes nicht $\mathrm{zu}$ finden vermag; so lange wir vom Protoplasma fast nicht mehr wissen, als dass es in verschiedenen Zellen sehr verschieden beschaffen $z \mathfrak{u}$ sein scheint.

Während ich ein näheres Eingehen auf die übrige Literatur auf spätere Gelegenheit verschiebe, möchte ich doch hier schon vorläufig zu den interessanten Resultaten $\mathrm{Sch}$ walbe's über die Kerne der Ganglienzellen (8) Stellung nehmen, die von den hier citirten jedenfalls am sorgfältigsten auf die allgemeine Seite der Sache eingehen. Schwal be beschreibt die kleinsten, jüngsten Ganglienzellenkerne als "scheinbar aus einer gleichmässig vertheilten, granulirten Masse bestehend ", die "wahrscheinlich auf eine netzförmige Structur zurückzuführen sei« - und es wird diese, wie weiter unten ausgesprochen ist, als gleichbedeutend mit einer Durchsetzung mit kleinen Vacuolen angesehen.

Indem diese Vacuolen wachsen, sollen nach Schwalbe die Zwischenwände immer mehr ausgedehnt und rareficirt werden, bis sie auf die Kernwand, die Nucleolen und die geringen Bruchstücke von Netzfäden reducirt sind, die er in den ausgewachsenen Kernen gefunden hat. An diesen Letzteren soll der bei weitem grösste Theil des Inhalts klare Masse sein. Der ganze Process sei also eine Vacuolisirung. Ich habe nun mit Bezug darauf zu erinnern, dass man alle die hier besprochenen Arten von Kernen, in denen allen dichte Netze zu sehen sind, ja unmöglich als junge ansehen kann ${ }^{1}$ ); und dass also, wenn man die netzförmige Structur einmal zugiebt, wie es Schwalbe ja thut, auch die Annahme gemacht werden muss, dass die Netze auch im ausgewachsenen Kern eine perman ente oder doch nur zeitweilig unterbrochene Rolle haben. Ob sie

1) Wenn ich im Eingang sagte, dass junge Thiere am vortheilhaftesten seien, so bezieht sich dies nur auf die Fibrillenarmuth bei jenen. Die von mir untersuchten jungen Thiere, von denen ich nur drei hatte, waren schon über fingerlang und übrigens stammen die meisten Abbildungen von ganz ausgewachsenen, bei denen sich die Kerne ganz wie bei jungen verhalten. 
nun allen Kernen zukommen, ist damit nicht entschieden. Wenn Schwalbe an den Ganglienzellen nur Bruchstücke und theilweise gar nichts daran sah, so kann das zwei Gründe haben. Entweder fehlen sie dieser Kernart wirklich, oder sie sind im frischen Zustand hier zu blass um gesehen zu werden; dass das Letztere möglich ist, dafür giebt meine obige Beschreibung ja hinreichende Belege. Darum dürfte es sich wohl nicht empfehlen, dass man, wie Schwalbe, einen derartigen negativen Befund an einer Zellenart benutzt, um darauf eine Theorie über die Bedeutung der Nucleolen zu gründen; um so weniger, da die oben besprochenen Färbungsresultate ergeben, dass die Kernkörperchen jedenfalls nicht bloss lokale Anhäufungen derNetzsubstanz, sondern etwas in dieser Differenzirtes sind, oder mindestens in vielen Fällen sein können. Zusammenhänge von Fortsätzen des Kernkörperchens (d.i. also offenbar Balken der hier beschriebenen Geruiste) mit der Kernwand scheint Schwalbe nicht constatirt zu haben (vgl. l. c. p. 29 und 37), und anzunehmen, dass dieselben im Innern des Kerns zugespitzt enden (p. 29). Ich muss dem gegenüber nach dem Obigen solche Zusammenhänge als das Regelmässige betrachten, obwohl sie oft, namentlich am lebenden Object, wegen ihrer Zartheit nicht zu sehen sind. Dagegen komme ich mit $S \mathrm{ch}$ wa lbe darin ganz überein, dass Zusammenhänge der Balken durch die Kernwand mit eventuellen Netzen im Plasma der Zellen auch mir unerfindlich waren.

Ein Punkt, der mir sehr auffällig gewesen ist, muss zum Schluss noch erwähnt werden. Bei keiner der hier beschriebenen Behandlungsweisen der Salamanderblase gelingt es mir bis jetzt, in den Kernen, die doch gerade durch ihre Grösse gute Chancen dafür boten, die von Eimer entdeckte Anordnung kleiner Körner im Kern nach concentrischen Zonen zu sehen. Ich kenne diese Bilder nach Eimer'schen und eigenen Präparaten (Osmiumsänre, Gold, Pikrokarmin-Essigsäure) gut genug, um mit Eimer von ihrem häufigen Vorkommen überzeugt zu sein, sie sind ja auch durch Auerbach bestätigt, der ihnen freilich eine andere Deutung als der Entdecker giebt. Dass man sie so, wie sie sind, als ein Strukturverhältniss des lebenden Kerns anzusehen habe, scheint auch mir durch nichts erwiesen, aber es kann ihrem Auftreten bei bestimmter Behandlung jedenfalls doch eine natürliche Bedingung im Kern zu Grunde liegen. Wenn dem so ist, so werden die weiter erforderlichen Bedingungen durch die hier gebrauchten Reagentien nicht erfüllt, und es wird 
auf ferneres Probiren ankommen, um über die Sache ein Urtheil zu gewinnen.

Im Anhang notire ich vorläufig noch einige andere Punkte hinsichtlich der Bestandtheile der Blasenwand, die ich weiter zu untersuchen im Begriff bin.

Die organischen Muskelzellen der Blase von Salamandra zeigen sowohl lebend, als nach Behandlung mit Reagentien die Längsstreifung, die ja schon anderweitig (A r nold) bekannt ist, in einer vorzüglichen Deutlichkeit.

Diese Muskelzellen sind, wo sie einzeln laufen, sehr vielfach nicht spindelig, sondern 3-4- bis selbst 6-fach verästelt (Fig. 4 u. 5). Ich zweifle darum nicht mehr daran, dass die verästelten Fasern in der Wand kleiner Lymphgefässe, die ich kürzlich beschrieb (ds. Arch. Band XII), in der That Muskelfasern sind. - Das Vorkommen von dreifach getheilten Muskelzellen ist ubrigens auch bereits von Klebs ${ }^{1}$ ) und Arnold (Strickers Handbuch) an der Blase des Frosches beschrieben worden. Ich muss aber zweifeln, ob Arnold damit dieselben Dinge im Auge gehabt hat, die ich hier meine, da er angiebt, die trichotomischen Fasern fänden sich vorzugsweise an den Theilungsstellen der grösseren Bündel, während sie, wie gesagt, vielmehr grade als frei verlaufende Fasern in den Maschen vorkommen, die von den Netzen der gröberen Bündel gelassen werden und zwar sowohl bei Rana wie bei Salamandra. Ich bemerke, dass man diese einzeln laufenden Fasern nur dann sicher an erhärteten Präparaten erkennen und verfolgen kann, wenn man ausser dem Epithel auch das Endothel der Blase entfernt hat, oder auch an dem sehr durchsichtigen lebenden Object; ohne diese Massregeln wird man sie meist mit verästelten Bindesubstanzzellen verwechseln, jedenfalls nicht sicher von solchen unterscheiden können.

Ueber die Nerven der Muskeln und die übrigen orientirte ich mich mittelst der Löwit'schen Goldmethode ${ }^{2}$ ), die für das Object in der That bis jetzt unübertrefflich ist. Für dieMuskelnerven finde ich durchaus bestätigt, was $L \ddot{o} w i t$ a. a. 0 . mitgetheilt hat und was ich zum Theil schon an dessen Präparaten gesehen hatte. Es scheinen mir aber ausser den für die Muskeln bestimmten noch andere in der Blasenwand nachweisbar.

1) Virchow's Arch. Bd. 32, 1865, p. 174.

2) Löwit, Ueber die Nerven der glatten Musculatur. Wiener Sitzungsbericht 1875 . 
Ueber das Epithel erwähne ich, dass darin constant in grösseren Abständen Zellen von ziemlich gleichmässiger Vertheilung, starkem Glanz und gelblicher Farbe vorkommen, die eigenartige Reaktionen zeigen.

Die Fibrillenbündel der Bindesubstanz sind sehr fein, aber leimgebend. Die Bindesubstanzzellen (Fig. 4) zeigen sich als vielfach verästelte und zusammenhängende Figuren. Dem entspricht das Goldpräparat. Es muss aber natürlich offen bleiben, ob dies die ganzen Zellen sind, oder nur verzweigte Plasmamassen, die auf je einer Zellplatte lagern.

\section{Literatur, auf deren Nummern im Text verwiesen ist.}

(Nach der Reihenfolge der Publikation.)

1. N. Kleinenberg, Hydra, pag. 41.

2. Th. Eimer, Die Schnauze des Maulwurfs als Tastwerkzeug. Archiv f. mikr. Anat. Bd. 7, pag. 189, 1870 und: Ueber die Eier der Reptilien, ebenda irn folg. Bande. (S. Nr. 4).

3. C. Heitzmann, Untersuchungen über das Protoplasma, I u. II, Wiener Sitzungsberichte, 17. April u. 23. Mai 1873.

4. L. A uerbach, Organologische Studien. Heft I, 1874.

(Nr. 2 und 4 enthalten nichts über Gerüste im Kern.)

5. W. Fle m ming, Studien in der Entwicklungsgeschichte der Najaden. Wiener Sitzungsberichte, 4. Febr. 1875. Mai 1875.

6. Ed. Strasburger, Ueber Zellbildung und Zelltheilung. Jena

7. C. Frommann, Zur Lehre von der Struktur der Zellen. Jenaische Zeitschr. f. Naturw. B. 11, S. 180.

8. G. Schwalbe, Bemerkungen über die Kerne der Ganglienzellen. Jenaische Zeitschrift für Naturwissenschaft, Mai 1875.

9. O. Hertwig, Beiträge zur Kenntniss der Bildung, Befruchtung und Theilung des thierischen Eies. Habilit.-Schrift, Leipzig. August 1875.

10. Edouard van Beneden, La maturation de l'oeuf, la fécondation et les premières phases du développement embryonnaire des Mammifères. Bull. de l'acad. Royale de Belgique, 2. Sér. t. 40, 1875.

11. Derselbe. Contributions à l'histoire de la vésicule germinative et du premier noyau embryonnaire. Ebenda, janv. 1876.

12. W. Mayzel, Ueber eigenthümliche Vorgänge bei der Theilung der Kerne in Epithelialzellen. Centralbl. f. d. med. Wiss. 1875. Nr. 50.

13. C. Kupffer, Ueber Differenzirung des Protoplasma an den Zellen thierischer Gewebe. Vortrag, gehalten im physiologischen Verein zu Kiel. 
Schriften des naturwiss. Vereins für Schleswig-Holstein; Heft 3, S. 222; sowie desselben Angaben über Struktur der Speicheldrüsenzellen von Blatta in: Beitr. z. Anat. u. Physiologie, als Festgabe für Carl Iudwig, 1875.

14. O. Bütschli, Studien über die ersten Entwicklungserscheinungen der Eizelle. Frankf. 1876.

15. Richard Hertwig, Beiträge zu einer einheitlichen Auffassung der verschiedenen Kernformen. Morphol. Jahrbuch 1876, Bd. 2. H. 1, 1876. p. 63.

\section{Erklärang der Abbildungen anf Tafel XLII.}

Fig. 1 a-d. Kerne der lebenden Harnblasenwand von Salamandra maculata, ohne Zusatz, mit Hartnack Syst. 8 u. 9. à imm. u. Oc. 3 gezeichnet.

a und $\mathrm{a}^{\prime}$ Bindesubstanzkerne,

b Endothelketroe der Aussenfläche,

c Muskelkern,

d Nervenkern.

Fig. 1 e. Kern einer Knorpelzelle vom Femurkopf von Salamandra, relativ dicker Schnitt, ohne Zusatz, etwa 20 Sekunden nach dem Abschneiden beobachtet.

Fig. 2. Epithelkern der frischen Blasenwand unmittelbar nach Zusatz von verdünnter Essigsäure und Glycerin. 9. 3. halb eing. Tub.

(Für diese Fig. ist zu bemerken, dass die kleinen hellen, dunkelcontourirt gezeichneten Kreise nicht Nucleolen, sondern optische Querschnitte der Balken des Kerngerüstes darstellen. Ein grösserer Nucleolus und drei Nebennucleolen sind zum Unterschied davon in der Abbildung ganz dunkel angegeben. 9. 3.

Fig. 3. Ein Bindegewebskel a der Blase nach Anfspritzung mit dünner Osmiumsäure und Färbung mit Pikrocarmin. Die rothgefärbte Substanz ist von der Kernmembran an einigen Stellen zurückgezogen. Zwei grössere und ein kleinerer Nucleolus sind deutlich. Von Netzen nur Andeutungen. 9. 3.

Fig. 4. Chromsaures Kali, Hämatoxylinfärbung. Ein Stück der Blasenwand nach Abpinselung des Epithels, die Fibrillen sind weggelassen. (Concentration des Chromkali 2 p. c.)

M Muskelzellen, eine davon trichotomisch, e Endothelkerne, b Bindesubstanzzellen mit ihren Kernen. An den Muskelzellen deutliche Längsatreifung. In den Kernen die Gerüste stark blau gefärbt, die übrige Substanz blasser, doch stärker wie das Plasma der Zellen. Fin Muskelkern, ein Endothelkern und ein Bindesubstanzkern (bei ***) sind blasser gefärbt und haben blassere Netze, wie die übrigen. 8. 3 eing. Tubus.

Fig. 5. Mitteltheil einer $6 \mathrm{fach}$ verästelten Muskelzelle mit deutlicher Fi- 
brillenstructur, gleiche Behandlung wie Fig. 4, Imm. 10. Oc. 3. eing. Tub.

Fig. 6. Endothelkern, ebenso behande]t, Imm. 10. Oc. 3, etwas eing. Tubus.

[In beiden Kernen sind keine Kernkörperchen zu unterscheiden; die dunkel gezeichneten Punkte entsprechen optischen Querschnitten der blau gefärbten Netzbalken.]

Fig. 7. Behandlung mit concentrirtem Kali bichromicum und Carminammoniak. A Muskelkern, b Epithelkern mit dem angrenzenden Plasma der Zellen, das reticulirt aussieht. a: 8, 3. b: 10 à imm. 3. An den Kernen ist das Netzwerk und die Kernwand ohne Carminfarbe geblieben, blassgelb (hier hell dargestellt), während der übrige Kerninhalt roseuroth ist (dunkler gezeichnet). (Bei vorheriger Anwendung von schwächerem Chromkali kann man auch das Netzwerk und die Membran röthen.)

Kernkörperchen sind auch hier wieder richt deutlich zu sehen, nur optische Querschnitte von Balken.

Fig. 8. Chromsäure-Carmin. Endothelkern und Bindesubstanzkern. Grössere und kleinere Nucleolen sind deutlich gefärbt sichtbar, auch die Membran und das Netzwerk hat Carminfarbe. 8. 3. eing. Tub.

Fig. 9. Bindesubstanz-, Epithel- und Muskelkerne, nach Hermann'scher Anilinbehandlung (Alkohol, Safranin, Alkohol, Nelkenöl).

In dem Netzwerk, das blass rosenroth geblieben ist, sind crass gefärbte Stellen, in den verschiedenen Kernen von ganz verschiedener Menge und Vertheilung, welche nicht alle Nucleolen sein können, weil so viele grössere Nucieolen, wie z. B. in a, b und d, mit andern Methoden nicht darstellbar sind. 7. 3.

Nach Rückbehandlung (Alkohol, Wasser, dann Essigsäure) sind in den sämmtlichen Kernen die dunklen Anilinflecken und selbst die Andeutung ihrer Stellen verschwunden, die Kerne gewähren nun ganzdie Bilder wie nach blosser Essigsäurebehandlung (s. Fig. 2).

Fig. 10. Kerne der äusseren Körnerschicht der Retina, Katze, nach Herma n n'scher Anilinbehandlung. Die Querschichtenstruktur der Kerne drückt sich in scharfem Farbenunterschied der abwechselnden Schichten aus.

Die mit Reagentien behandelten Präparate sind, so weit darüber nichts Besonderes bemerkt wurde, in Wasser untersucht und conservirt, da Glycerin oder ätherische Oele die Gerüste weniger deutlich lassen. Ich bemerke noch, dass in der Schärfe der Netzzeichnungen nichts übertrieben, sondern darin möglichst genau dem Präparat gefolgt wurde.

(In einigen Figuren, besonders 1 und 8, sind die Netzbalken 3. Th. im Stich allzu zart und dünn ausgefallen.) 


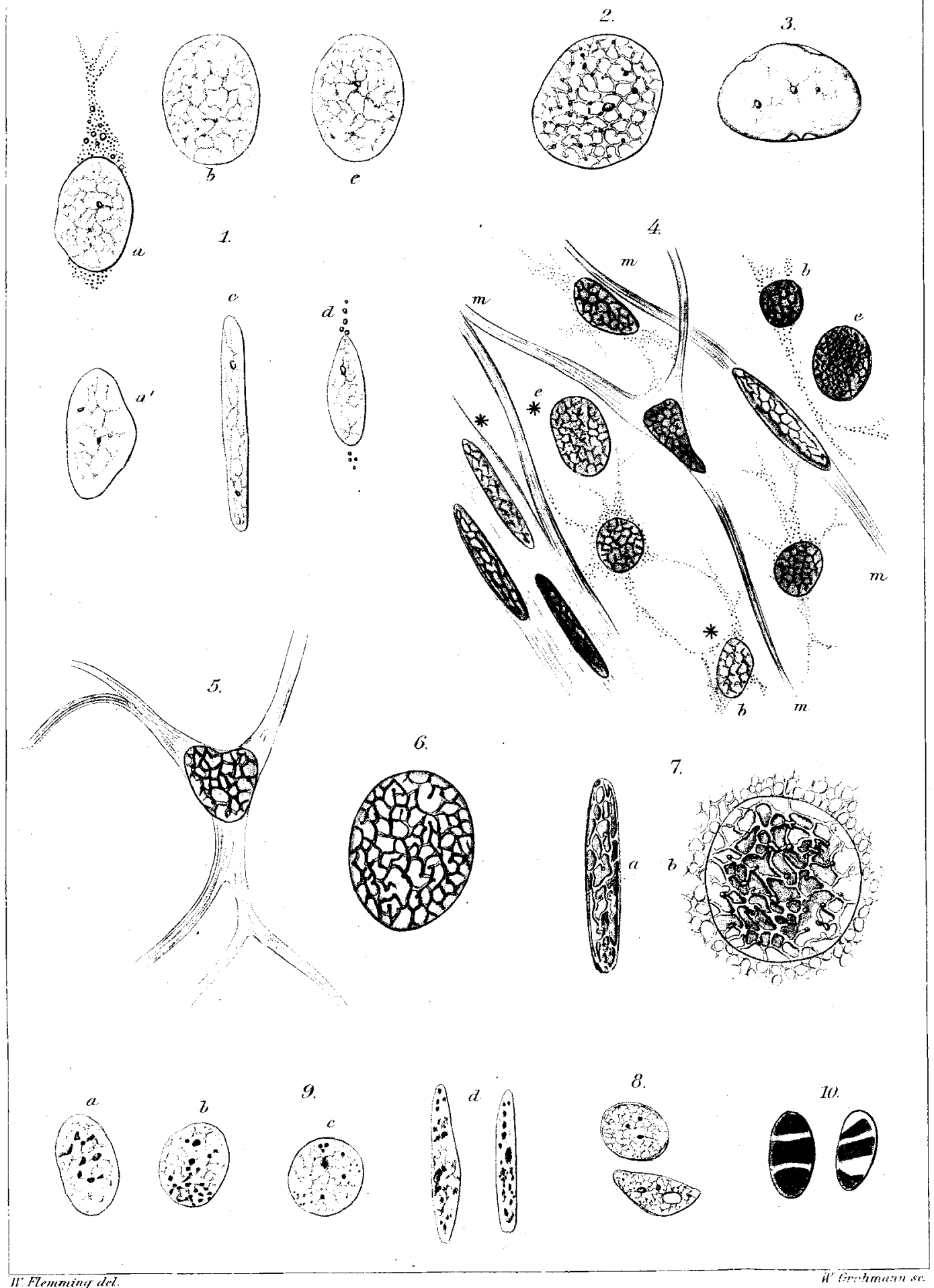

